\title{
La percepción de la reputación corporativa de los stakeholders de una empresa de comunicación: cómo afecta a Televisió de Catalunya comunicar una acción de responsabilidad social corporativa
}

\section{Jaime Alberto Orozco-Toro ${ }^{1}$ Carme Ferré-Pavia ${ }^{2}$}

Recibido: 2016-02-03

Enviado a pares: 2016-02-09
Aprobado por pares: 2016-03-28

Aceptado: 2016-04-13

DOI: 10.5294/pacla.2017.20.2.8

Para citar este artículo / to reference this article / para citar este artigo Orozco-Toro, J. A. y Ferré-Pavia, C. (2017). La percepción de la reputación corporativa de los stakeholders de una empresa de comunicación: cómo afecta a Televisió de Catalunya comunicar una acción de responsabilidad social corporativa. Palabra Clave, 20(2), 473-505. DOI: 10.5294/pacla.2017.20.2.8

\section{Resumen}

Esta investigación se centra en determinar cómo la comunicación de la responsabilidad social corporativa incide en la percepción de reputación corporativa de las empresas de comunicación, tomando como objeto de análisis toda su cadena de valor, lo cual es novedoso en el campo. En este sentido, se asume la comunicación de la responsabilidad social corporativa como parte del compromiso adquirido con todos los stakeholders. El trabajo empírico toma como caso La Marató de TV3 (recogida de fondos para investigación médica del canal autonómico público de Cataluña, España),

orcid.org/0000-0002-8152-7474. Universidad Pontificia Bolivariana. Colombia. jaime.orozco@upb.edu.co

2 orcid.org/0000-0002-7258-6376. Universidad Autónoma de Barcelona. España. Carme.Ferre@uab.cat 
y cómo esta actividad de solidaridad y comunicación influye en la imagen y la reputación corporativa del canal. La herramienta metodológica aplicada es la encuesta. Se han obtenido 463 encuestas realizadas a los 7 grupos de interés de toda su cadena de valor. Los resultados se expresan con perspectiva cuantitativa y cualitativa y permiten dar cuenta de que la responsabilidad social corporativa tiene una relevante incidencia en la imagen y la reputación corporativa de la marca, que se convierte en una condición fundamental para mantener los vínculos con cada uno de sus stakeholders.

\section{Palabras clave}

Responsabilidad social corporativa; reputación corporativa; stakeholders; empresas de comunicación; La Marató de TV3 (Fuente: Tesauro de la Unesco). 


\section{The Perception of the Corporate Reputation of the Stakeholders from a Communication Company: How Televisió de Catalunya is Affected by Communicating a Corporate Social Responsibility Action}

\section{Abstract}

This research focuses on how the communication of corporate social responsibility affects the perception of corporate reputation of communication companies, taking the entire value chain as an object of analysis, which is novel in the field. In this sense, the communication of corporate social responsibility is assumed as part of the commitment to all stakeholders. The empirical work takes as a case La Marató de TV3 (fundraising for medical research from the autonomous public channel of Catalonia-Spain), and how this solidarity and communication activity influences the image and corporate reputation of the channel. The methodological tool applied is the survey. A total of 463 surveys were carried out in the 7 interest groups throughout their entire value chain. The results are expressed with a quantitative and qualitative perspective and allow to evidence that corporate social responsibility has a relevant impact on the image and corporate reputation of the brand, which becomes a fundamental condition to maintain the links with every stakeholder.

\section{Keywords}

Corporate social responsibility; corporate reputation; stakeholders; communication companies; La Marató de TV3 (Source: Unesco Thesaurus). 


\section{A percepção da reputação corporativa dos stakeholders de uma empresa de comunicação: como afeta a Televisió de Catalunya comunicar uma ação de responsabilidade social corporativa}

\section{Resumo}

O foco desta pesquisa é determinar como a comunicação da responsabilidade social corporativa incide na percepção de reputação corporativa das empresas de comunicação, tomando como objeto de análise toda a sua cadeia de valor, o qual é novo neste campo. Neste sentido, se assume a comunicação da responsabilidade social corporativa como parte do compromisso adquirido com todos os stakeholders. $\mathrm{O}$ trabalho empírico toma como caso La Marató de TV3 (coleta de fundos para pesquisa médica do canal autonômico público da Catalunha-Espanha), e como esta atividade de solidariedade e comunicação influi na imagem e a reputação corporativa do canal. A ferramenta metodológica aplicada é a enquete. Foram obtidas 463 enquetes realizadas aos 7 grupos de interesse de toda a sua cadeia de valor. Os resultados se expressam com perspectiva quantitativa e qualitativa e permitem perceber que a responsabilidade social corporativa tem uma relevante incidência na imagem e na reputação corporativa da marca, que se transforma em uma condição fundamental para manter os vínculos com cada um de seus stakeholders.

\section{Palavras-chave}

Responsabilidade social corporativa; reputação corporativa; stakeholders; empresas de comunicação; La Marató de TV3 (Fonte: Tesauro da Unesco). 


\section{Introducción}

Los estudios que relacionan la responsabilidad social corporativa (RSC) con la economía, la estrategia empresarial o el marketing, entre otros, son numerosos, en especial en los países anglosajones. Sin embargo, aquellos sobre la comunicación de la RSC son menos prolíficos. Más aún, si se quiere indagar sobre el vínculo entre la comunicación de la RSC y sus efectos en la reputación corporativa ( $\mathrm{RC}$ ), las investigaciones son más que escasas. No son recurrentes estudios que permitan exponer la forma en que la comunicación de la RSC afecta la RC, y aún menos si nos suscribimos a toda la cadena de valor (todos los grupos de interés que hacen parte de la organización y están afectados por sus actividades).

Hasta el momento, las exploraciones realizadas sobre la incidencia de la RSC en la RC no son concluyentes, incluso en algunos casos son contradictorias. Quizá sea porque no se ha abordado toda la cadena de valor. Si bien es cierto que los stakeholders cobran cada vez más importancia para las empresas, la mayoría de los procesos investigativos se centran en empleados y usuarios. Dadas tales condiciones, esta investigación se enfoca en la manera en que la RSC afecta a la RC, desde las percepciones de todos sus stakeholders. Se toma como caso de análisis La Marató de Televisió de Catalunya-TV3, la cadena de televisión pública autonómica de Cataluña (España), actividad de recogida de fondos para la investigación médica.

La Marató de TV3 fue la primera maratón televisiva de España, celebrada ininterrumpidamente desde 1992. Esta veteranía en las celebraciones le da credibilidad. Los telemaratones, a veces, son cuestionados como caridad espectacularizada, pero el de TV3 lleva veintitrés años implicando a la sociedad catalana. Por ejemplo, en la edición celebrada en 2014, dedicada a las enfermedades cardiovasculares, se superaron los 11 millones de euros de recaudación y siguieron el programa más de 400000 personas, con una cuota de pantalla de $19.5 \%$ (Fundació La Marató de TV3, 2015). 


\section{Marco teórico}

\section{La investigación de la responsabilidad social corporativa}

Con el advenimiento de la RSC, las investigaciones que proponen analizar su incidencia están a la orden del día. Además, una gran variedad de estos estudios vinculan aspectos esenciales de la estrategia de la compañía, con la intención clara y marcada de indagar, en especial, si la RSC ejerce un efecto específico o no.

Se pueden citar estudios empíricos, documentales, descriptivos, de casos, análisis de contenidos o de memorias anuales, entre muchos otros. Incluso, desde las condiciones específicas de estos, se localizan investigaciones sobre la RSC y la respuesta del consumidor (Du, Bhattacharya y Sen, 2010; Öberseder, Schlegelmilch y Murphy, 2013; Costa y Menichini, 2013), la RSC y su relación con la gestión empresarial (Lindgreen et al., 2012; Tang, Hull y Rothenberg, 2012; Lee, Park y Lee, 2013), el marketing de la RSC (Laczniak y Murphy, 2012; Torelli, Monga y Kaikati, 2012; Charlo, Moya y Muñoz, 2013), la RSC en empresas de comunicación (Orozco y Ferré Pavia, 2011; Ingenhoff y Koelling, 2012; Fernández, 2013), entre otras.

Por su parte, las investigaciones relacionadas con la imagen y la RSC han estado enmarcadas tradicionalmente por un paradigma que para algunos autores (Simic y Belliu, 2011; Arendt y Brettel, 2010; Bigné, Currás y Aldás, 2012) parece incuestionable: la idea de que la RSC ayuda a mejorar la imagen de las empresas. Sin embargo, otros indican que la RSC se maneja dentro de los departamentos de comunicación más como una necesidad que como una oportunidad (Olcese, 2011). Desde esta óptica, la relación de las acciones de RSC con la imagen deberá ser entendida como una oportunidad para el diálogo con los grupos interesados, y no solo como un depósito de reserva de una imagen positiva que ayude a suavizar una crisis.

Aunque la RSC puede mejorar la imagen de una compañía, las acciones que se implementen deben ser creadas como parte de una estrategia integral de marca, al contrario el impacto sobre los stakeholders será mínimo, incluso contraproducente (Simic y Belliu, 2001). En las empresas de 
comunicación, es uno de los ámbitos empresariales donde menos acciones de RSC se detectan. Una de las formas para comprobarlo es verificar las empresas adheridas al Pacto Mundial de las Naciones Unidas: el renglón perteneciente a las empresas de comunicación es uno de los que menos participación tiene (Ferré Pavia y Orozco, 2011, p. 101).

De todo ello queda una conclusión principal, y es que muy a pesar de los avances alcanzados, aún existen muchos aspectos por abordar, sobre todo, en lo relacionado con las empresas de comunicación, la RC y las percepciones que tienen todos los stakeholders de su cadena de valor de las iniciativas que desarrollan en la RSC.

\section{Estrategias de comunicación de la responsabilidad social corporativa}

Aunque los resultados de las investigaciones con relación a la comunicación de la RSC sean contradictorios, lo interesante está en advertir que esta actividad cobra cada vez mayor importancia, dado que investigadores y directivos han entendido la enorme relevancia de un vínculo transparente con los stakeholders.

Las estrategias de comunicación institucional o comercial de una organización difieren de las estrategias de comunicación de la RSC. Las bases de la publicidad y la comunicación comercial de la marca que han definido importantes autores (Aaker, 2002; Sanz de la Tajada, 1996; Van Riel, 1997; Cerviño, 2002; Borrini, 2006) están mediadas por una relación directa con los públicos objetivos a través de la promoción de productos y servicios. Estos han sido tradicionalmente los que han acaparado el espectro de la comunicación entre las marcas y sus consumidores. En contraposición, la comunicación de la RSC está orientada a la identidad y a los valores corporativos que el ente comparte con todos sus grupos de interés.

Además de la complejidad de comunicar la RSC, uno de los mayores retos a los que se enfrentan los encargados de estas estrategias es la decisión de si es correcto o no difundir las acciones de RSC y, posteriormente, establecer la intensidad de dicho vínculo con los stakeholders. La discusión de comunicar o no tiene menores implicaciones, pues instituciones públicas y 
privadas, como el Global Compact de las Naciones Unidas, el Libro Verde de la Comisión Europea o el Global Reporting Initiative, recomiendan dar a conocer los esfuerzos en la RSC que desarrollan las empresas. El meollo de la situación radica en definir la intensidad de esta relación. Para algunos autores (Miralles, 2006; Ráfols, 2007; Ramiro, 2009; Doane, 2010; Mainwaring, 2011), una comunicación excesiva de la RSC ha sido interpretada por los públicos como maquillaje (Villagra, 2007). Aunque no existe una fórmula mágica o una medida estándar que determine la intensidad de la comunicación, la clave está en la realización de una comunicación transparente (Epstein y Birchard, 2001).

\section{El concepto de reputación corporativa}

La RC no es un concepto nuevo para la empresa, si bien es cierto que los mayores avances en investigación y en su análisis se han generado en los últimos años. Así lo corrobora Larkin al destacar que "la investigación académica está aún en la infancia [La traducción es nuestra]” (2003, p. 40).

Aunque es posible que anteriormente no se diera a la RC el valor que tiene, hoy los intangibles, activos que producen beneficios a la empresa, adquieren una gran importancia debido a que "la marca es la visible punta del iceberg [... pero la reputación permanece escondida al acecho bajo la superficie” (Hannington, 2006, p. 37). Y no solo cobra importancia la RC por haberse convertido en un valor fundamental en la gestión de los intangibles (Pérez Serrano, 2010), sino por los vínculos que tiene con la identidad, la imagen, la marca, inclusive por ser considerada como "el eslabón perdido entre los negocios y la ética [La traducción es nuestra]" (Jackson, 2004, p. 86).

A diferencia de lo que ocurre con el concepto de RSC, en el que existe una profusión conceptual y terminológica que complica su desarrollo, en las teorías de la RC existe consenso sobre su significado. Algunos de los autores más importantes (Sanz de la Tajada, 1994; Fombrun, 1996; Van Riel, 1997; Dowling, 2001; Argenti y Forman, 2002; Quevedo, 2003; Alsop, 2004; Freeman, 2006; Álvarez, 2008; Capriotti, 2009; Firestein, 2009; Doorley y García, 2011; Earl y Waddington 2012) coinciden en aspectos determinantes, lo cual permite un análisis mucho más fluido. 
Por ejemplo, Alsop (2004) advierte que la RC está basada en percepciones de la gente y que, aunque tarda años en construirse, puede ser arruinada en un instante. Asimismo, se concibe la RC como percepciones colectivas sobre una marca (Lange, Lee y Dai, 2011), incluso la percepción llega a tener una alta importancia, ya que se considera como "la fuerza motora entre el éxito o el fracaso en la gestión [La traducción es nuestra]" (Larkin, 2003, p. 4).

\section{La investigación de la reputación corporativa}

Que la RC sea una teoría en desarrollo hace que se dé una evolución epistemológica. Lo importante no ha sido la aplicación de un modelo empírico, sino primero sentar las bases de su conceptualización teórica y luego la propuesta de índices para su evaluación.

Hoy día, hay problemas reconocidos con los índices que miden la RC empresarial, además, se promueven herramientas más específicas que se amolden a líneas económicas que segmenten los grupos de interés. Desde la perspectiva de estos grupos, la mayor preocupación de los directivos de las compañías que están relacionados con el tema de la RC es identificar su impacto en los stakeholders, pero, en especial, en los consumidores o usuarios. Teniendo presentes las expectativas del sector empresarial, la mayoría de las investigaciones se han enfocado en un solo grupo de interés, sin tener presente la relevancia de la RC en toda la cadena de valor.

Si bien las empresas de comunicación, en especial, los medios de comunicación, han tenido una injerencia directa sobre la reputación de las empresas, en este sector han sido menos importantes las acciones relacionadas con la RC. Asimismo, la proliferación de iniciativas de la RSC ha generado un incremento en las investigaciones en las que se intenta conocer su relación e impacto en la RC. Algunas de las más importantes investigaciones vinculadas a la $\mathrm{RC}$ han sido la relación entre los estudios de la RC y los stakeholders (Mishina, Block y Mannor, 2012; Hillenbrand, Money y Ghobadian, 2013; Helm y Tolsdorf, 2013), el vínculo entre marketing y la RC (García, 2002; Alloza, 2005; Olabe, 2012; Delgado, Quevedo y Díez, 2013) y la implicación entre las acciones de la RSC yla RC (Capriotti, 2007; 
Castaldo et al., 2009; Orozco y Roca, 2011; Kim, 2011; Saeedniaa y Sohanib, 2013; Martínez, Pérez y Rodríguez, 2014).

\section{La reputación social corporativa y la reputación corporativa en empresas de comunicación}

Dos nuevos paradigmas de la comunicación vienen formándose en los últimos años. En primer lugar, la preocupación de las empresas de comunicación y de los stakeholders por temas relacionados con la RSC, y en segundo término, la nueva relevancia que se le da a la construcción de reputación en las empresas de comunicación. Sin embargo, el ámbito de la investigación sobre la relación RSC y RC en las empresas de comunicación se encuentra en una situación de desatención.

Las empresas mediáticas desempeñan un papel líder en estos nuevos paradigmas, ya que reciben la afluencia de comunicación proveniente de las iniciativas de la RSC. Además son las garantes del vínculo creador de la RC a través del contacto con los stakeholders. En este momento, los valores promulgados por la ética, la RSC y la RC deben ser utilizados como elemento de cohesión entre las organizaciones y sus grupos de interés. Sin embargo, la preocupación más reiterativa de muchos investigadores es la postura de empresas donde se implementan códigos éticos como estrategia que proporcione una imagen positiva en el mercado (Sánchez, 2001; Preciado-Hoyos, 2015). Este hecho no solo es menester de las empresas de comunicación, sino que ha sido observado con mucha atención desde que la RSC comenzó a ser utilizada como "lavado de cara”. En las empresas de comunicación, la mayor responsabilidad había sido suscrita a entregar un producto o servicio de calidad y cumplir con las obligaciones ante los accionistas. Las responsabilidades con respecto a otros grupos, como los proveedores, los anunciantes o las instituciones, quedaban relegadas a un segundo plano.

Las responsabilidades de las empresas de comunicación se amplían no solo por la irrupción de la teoría de los stakeholders, sino también por los intereses generados en las acciones de la RSC. Los viejos objetivos de informar, formar, entretener e influir han abierto un espacio a las nuevas responsabilidades permeadas por la $\mathrm{RSC}$. Al mismo tiempo, estas empre- 
sas deben cumplir con sus propias responsabilidades sociales y dar a conocer a la opinión pública lo que significa la RSC.

\section{Diseño metodológico}

La herramienta para analizar la percepción de la cadena de valor es una encuesta aleatoria a miembros de todos sus stakeholders, internos y externos, a los que se encuestó por canales distintos, como indica la tabla 1. Las preguntas refieren los indicadores de la RSC que se evalúan. Estas son cerradas (sí/no, no sabe) y abiertas con libertad de respuesta. Las preguntas formuladas son:

1. ¿Considera transparente la gestión que realiza TV3 con su iniciativa de La Marató?

2. ¿Las acciones que desarrolla La Marató contribuyen a mejorar la gestión empresarial de TV3?

3. ¿La iniciativa La Marató ayuda a mejorar la reputación de TV3?

4. ¿TV3 utiliza iniciativas como La Marató para mejorar su imagen de marca ante los proveedores?

5. ¿Considera que La Marató es una acción de RSC de TV3?

6. ¿Las acciones de La Marató ayudan a mejorar la relación de TV3 con los proveedores?

El análisis tanto cuantitativo (respuestas cerradas) como cualitativo (respuestas abiertas) ha cruzado la estricta respuesta vinculada al ámbito encuestado (por ejemplo relación) con un análisis de contenido que ha permitido fichar las recurrencias al mismo ámbito con palabras distintas (vínculo, implicación, identificación...).

Dado que los grupos de interés de TV3 no son homogéneos en cuanto a la medida de su universo, el nivel de respuesta de cada uno está directamente relacionado con su población total. Así, por ejemplo, la población más amplia es la de la Ciudadanía, pues se tiene en cuenta a los habitantes de Cataluña (7.5 millones de personas), mientras que los Clientes de TV3 eran 875. 


\section{Tabla 1. Ficha técnica del estudio empírico}

\begin{tabular}{|l|l|}
\hline Procedimiento metodológico & $\begin{array}{l}\text { Encuesta presencial aleatoria (ciudadanos y administración), encuesta a } \\
\text { través de correo electrónico (asociaciones y empresas), encuesta aleatoria } \\
\text { a voluntad a través de la intranet de TV3 (trabajadores) }\end{array}$ \\
\hline Tipo de preguntas planteadas & Abiertas y cerradas \\
\hline Población & Infinita \\
\hline Ámbito geográfico & Comunidad Autónoma de Cataluña, todas sus provincias \\
\hline Número de encuestas totales realizadas & 463 \\
\hline Número de stakeholders de cadena de valor & 7 \\
\hline Margen de error & $4.5 \%$ \\
\hline Nivel de confianza & $95 \%$ \\
\hline Fecha trabajo de campo & 2014 \\
\hline Tratamiento de la información & Informático, a través de Excel \\
\hline
\end{tabular}

\section{Tabla 2. Ficha técnica de la muestra de población encuestada}

\begin{tabular}{|c|c|c|c|c|}
\hline \multirow{3}{*}{ Stakeholders } & \multirow{3}{*}{$\begin{array}{l}\text { Número de } \\
\text { encuestas }\end{array}$} & \multicolumn{2}{|c|}{ Sexo (promedio) } & \multirow{3}{*}{$\begin{array}{c}\text { Promedio } \\
\text { de edad } \\
\text { (años) }\end{array}$} \\
\hline & & Hombres & Mujeres & \\
\hline & & \multicolumn{2}{|c|}{ (cifras en porcentaje) } & \\
\hline Empleados & 53 & 49 & 51 & 47 \\
\hline Clientes & 17 & 47 & 53 & 43 \\
\hline Proveedores & 31 & 62 & 38 & 45 \\
\hline Ciudadanía & 266 & 42 & 58 & 41 \\
\hline Organizaciones, instituciones y asociaciones & 55 & 51 & 49 & 43 \\
\hline Competencia y sector estratégico & 19 & 52 & 48 & 39 \\
\hline Instituciones públicas & 22 & 50 & 50 & 45 \\
\hline Total & 463 & 50.5 & 49.5 & 42 \\
\hline
\end{tabular}

\section{Resultados}

Los resultados de esta investigación vienen presentados por orden, en respuesta a cada pregunta de la encuesta, que, a su vez, remite a intangibles y ámbitos de la RSC. Estos han permitido tanto recolectar información de las percepciones de los siete grupos de interés que forman la cadena de valor de TV3, a través de la iniciativa solidaria La Marató, para evaluar la incidencia de la comunicación de la RSC en su RC. Estos se expresan con un doble resultado cuantitativo y cualitativo, que complementa las respuestas que podrían llevar a un análisis superficial. 


\section{La transparencia en la gestión de La Marató de TV3}

La primera pregunta solicita a los encuestados si consideran transparente la gestión que realiza TV3 a través de su iniciativa de La Marató (tabla 3).

\section{Tabla 3. Transparencia en la gestión de La Marató de TV3}

\begin{tabular}{|l|c|c|c|}
\hline \multicolumn{1}{|c|}{ Stakeholders } & $\begin{array}{c}\text { Sí } \\
\text { (cifras en porcentaje) }\end{array}$ & (cifras en porcentaje) & NS/NR \\
\hline Empleados & 94.3 & 3.7 & 2.0 \\
\hline Clientes & 100 & 0.0 & 0.0 \\
\hline Proveedores & 90.0 & 3.2 & 6.8 \\
\hline Ciudadanía & 74.0 & 13.9 & 12.1 \\
\hline Organizaciones, instituciones y asociaciones & 69.0 & 16.4 & 14.6 \\
\hline Competencia y sector estratégico & 89.4 & 5.3 & 5.3 \\
\hline Instituciones públicas & 86.3 & 13.7 & 0.0 \\
\hline Promedio & 86.2 & 8.0 & 5.8 \\
\hline
\end{tabular}

Una importante mayoría de los grupos de interés de TV3 evalúan como transparente su gestión. Los clientes internos (clientes, empleados y proveedores) consideran que TV3 es absolutamente transparente. Aunque el nivel más bajo de percepción de transparencia se puede apreciar en las organizaciones, instituciones y asociaciones con $69 \%$, aún es alto. Es destacable que sean los stakeholders los que tienen un mayor porcentaje de respuestas, en las que no consideran transparente la gestión de La Marató, ya que es precisamente el grupo de interés beneficiado con lo recaudado.

Desde una perspectiva más cualitativa, la percepción que se tiene sobre los procesos de accountability por parte de TV3 se divide entre quienes consideran completamente transparente la gestión, porque han recibido información y aquellos que aducen falta de transparencia:

Creo que sí que es transparente, dado que durante muchos meses antes desde el mismo medio de comunicación se hacen documentales y programas diversos relacionados con el tema que se debe tratar (Proveedores, directiva, 45 años).

No. Se desconoce cuáles son las partidas concretas y a qué programas van dirigidas. Se desconoce el número total de empresas y 
ciudadanos particulares que contribuyen y la media de aportación de cada tipo (Organizaciones, instituciones y asociaciones, licenciado, 42 años).

Aunque TV3 publica el destino de los fondos recaudados y las entidades a las que van dirigidos, aún hay stakeholders que desconocen estos esfuerzos de transparencia. La situación es comprensible, ya que las acciones de accountability se realizan, pero la información no siempre llega a todos.

Dependiendo del tipo de relación que tengan con La Marató y TV3, los stakeholders asumen una posición más o menos crítica. Muchos no solo se atreven a realizar críticas o alabar la gestión realizada, sino que proponen soluciones, en este caso la divulgación de los estados contables que permitan mayor transparencia:

La percepción que tenemos es que no hay intereses ocultos ni intención de ocultar nada. Quizá no estaría mal que en algún momento se hiciera una difusión amplia entre la ciudadanía de los costes que supone una iniciativa como esta (Organizaciones, instituciones y asociaciones, profesional, 67 años).

Como se observa, hay variedad de posiciones, pero en la mayoría de los casos los más críticos con TV3 son los stakeholders con menos información y contacto con la organización. Especialmente, la Ciudadanía es el colectivo donde más quejas se encuentran sobre la falta de transparencia emanada de la falta de información, a diferencia de los Empleados y los Proveedores, que sostienen una relación más estrecha con La Marató y el ente.

\section{La contribución de La Marató en la gestión empresarial de TV3}

Las diferencias de información de la cadena de valor se aprecian al reconocer que quienes más cercanía tienen con TV3 y con La Marató, como son Empleados, Clientes y Proveedores, aceptan en mayor proporción que esta iniciativa contribuye a mejorar la gestión. Sin embargo, los resultados son significativamente bajos en los siete grupos de interés. Desde esta perspectiva, al investigar la cadena de valor, las opiniones están bastante divididas. 


\section{Tabla 4. Contribución de La Marató en la gestión de TV3}

\begin{tabular}{|l|c|c|c|}
\hline \multicolumn{1}{|c|}{ Stakeholders } & $\begin{array}{c}\text { Sí } \\
\text { (cifras en porcentaje) }\end{array}$ & (cifras en porcentaje) & NS/NR \\
\hline Empleados & 39.6 & 52.8 & 7.6 \\
\hline Clientes & 70.5 & 29.5 & 0.0 \\
\hline Proveedores & 45.1 & 22.5 & 32.4 \\
\hline Ciudadanía & 42.8 & 25.5 & 31.7 \\
\hline Organizaciones, instituciones y asociaciones & 32.8 & 32.8 & 34.4 \\
\hline Competencia y sector estratégico & 36.8 & 47.3 & 15.9 \\
\hline Instituciones públicas & 36.3 & 22.7 & 41.0 \\
\hline Promedio & 43.4 & 33.3 & 23.3 \\
\hline
\end{tabular}

Quienes atribuyen un beneficio de La Marató en mejora de la gestión empresarial de TV3 consideran:

Sí contribuye porque es una muy buena práctica de gestión empresarial para una empresa de comunicación (Ciudadanía, licenciada, 22 años).

Es un exponente paradigmático de su vocación de servicio público. La clave del éxito de La Marató, en mi opinión, solo es posible porque la realiza TV3 (Clientes, director, 48 años).

También se presentan algunas opiniones que concluyen que La Marató no representa un beneficio en la gestión de TV3. Estas van desde quienes hablan de un lavado de cara hasta quienes solo ven La Marató como una acción puntual de una emisión de un programa de televisión:

Así hacen una limpieza ante La Caixa, Abertis, y otras empresas (Ciudadanía, licenciada, 33 años).

No, desde el momento que la gestión no está integrada ni centralizada como el resto de empresas del grupo (Empleados, licenciado, 41 años).

Además de las opiniones favorables o contrarias sobre los beneficios en la gestión empresarial de TV3, surgió un elemento que relaciona la labor realizada a través de La Marató con la obtención de publicidad, es 
decir, señalan que la gestión se ve beneficiada por los ingresos provenientes de los anunciantes:

Especialmente porque otorga a la marca una valoración muy positiva. TV3 = Marató $=$ algo bueno. La adhesión del público supone más televidentes y mejor posicionamiento para obtener publicidad (Competencia y sector estratégico, directora, 51 años).

Aunque en un principio la tasa de respuesta a esta pregunta tuvo un importante índice de stakeholders que no sabían o no respondían, se observa en las respuestas abiertas que sí que se conocen los efectos positivos y negativos que una acción de la RSC como La Marató puede ocasionar en la gestión de una empresa mediática.

\section{La incidencia de La Marató en la RC de TV3}

Una pregunta central de la investigación busca conocer la percepción de los stakeholders sobre cómo La Marató influye en la RC de la empresa analizada (tabla 5).

\section{Tabla 5. Incidencia de La Marató en la RC de TV3}

\begin{tabular}{|l|c|c|c|}
\hline \multicolumn{1}{|c|}{ Stakeholders } & $\begin{array}{c}\text { Sí } \\
\text { (cifras en porcentaje) }\end{array}$ & $\begin{array}{c}\text { No } \\
\text { (cifras en porcentaje) }\end{array}$ & NS/NR \\
\hline Empleados & 96.2 & 3.8 & 0.0 \\
\hline Clientes & 82.3 & 17.7 & 0.0 \\
\hline Proveedores & 93.4 & 3.3 & 3.3 \\
\hline Ciudadanía & 90.9 & 5.2 & 3.9 \\
\hline Organizaciones, instituciones y asociaciones & 90.9 & 5.6 & 3.5 \\
\hline Competencia y sector estratégico & 94.7 & 5.3 & 0.0 \\
\hline Instituciones públicas & 100 & 0.0 & 0.0 \\
\hline Promedio & 92.7 & 5.8 & 1.5 \\
\hline
\end{tabular}

En este aspecto, los resultados son contundentes debido a que no solo se obtuvo el porcentaje promedio más alto de toda la encuesta relacionando toda la cadena de valor, con $92.7 \%$ de los grupos que consideran que sí que hay incidencia en la RC, sino también es la pregunta con el porcentaje más bajo de NS/NR (1.5\%). Que toda la cadena valore de forma categórica la relación entre la RSC y su influencia en la $\mathrm{RC}$ permite revelar que 
no solo se tiene una valoración positiva de La Marató, sino de propuestas de la RSC como esta.

Es tan fuerte y arraigada la RC de TV3 que se pueden encontrar muchas manifestaciones de stakeholders que precisan que esta cadena de televisión no realiza La Marató con esta intención:

Aporta una imagen social y de preocupación por temas que afectan la vida del ciudadano. Se ve como una iniciativa seria, científica y social, no solo como un show para conseguir más audiencia (Empleados, redactor, 61 años).

No lo necesita, ya TV3 tiene una buena reputación (Ciudadanía, profesional, 41 años).

Los comentarios anteriores reconocen que, aunque La Marató mejora la RC de TV3, esta realmente posee ya un remanente importante que le permite gozar del reconocimiento de los stakeholders.

\section{Utilización de La Marató para mejorar la imagen de marca de TV3}

Los resultados de esta pregunta son interesantes, pero lo son aún más si se comparan con las respuestas anteriores sobre la incidencia de La Marató en la RC (tabla 6).

\section{Tabla 6. Beneficios de La Marató en la imagen de marca de TV3}

\begin{tabular}{|l|c|c|c|}
\hline \multicolumn{1}{|c|}{ Stakeholders } & $\begin{array}{c}\text { Sí } \\
\text { (cifras en porcentaje) }\end{array}$ & $\begin{array}{c}\text { No } \\
\text { (cifras en porcentaje) }\end{array}$ & NS/NR \\
\hline Empleados & 35.8 & 54.7 & 9.5 \\
\hline Clientes & 41.1 & 41.1 & 17.8 \\
\hline Proveedores & 41.9 & 45.1 & 13.0 \\
\hline Ciudadanía & 71.0 & 18.0 & 11.0 \\
\hline Organizaciones, instituciones y asociaciones & 72.8 & 9.1 & 18.1 \\
\hline Competencia y sector estratégico & 42.1 & 42.1 & 15.8 \\
\hline Instituciones públicas & 45.4 & 27.2 & 27.4 \\
\hline Promedio & 50.0 & 34.0 & 16.0 \\
\hline
\end{tabular}


Los stakeholders que más consideran que se mejora la imagen ante su propia condición como grupo de interés son las Organizaciones, instituciones y asociaciones, y es significativo igualmente que sean los Empleados quienes menos creen que La Marató genere una imagen positiva. La comparación entre las preguntas relacionadas con la imagen y la RC es necesaria, pues, las diferencias son marcadas. Refiriéndonos únicamente al promedio de cada uno, los stakeholders consideran que La Marató incide positivamente en la RC en $92.7 \%$, en contraposición con $50 \%$ que valora esta misma incidencia en la imagen ante los grupos de interés.

No obstante, este valor numérico se percibe de forma diferente al contrastar los comentarios de los grupos de interés: muchos encuestados expresan que TV3 realiza La Marató y recibe beneficios de su parte que redundan en una mejora de la imagen de marca, pero que no lo hace con ese objetivo. Consideran que sus acciones son transparentes, que no utilizan la RSC como un lavado de cara y, especialmente, que así TV3 utilizara La Marató con este fin no les importaría que fuera así.

La evidencia clara de que el uso de La Marató como parte de una herramienta para mejorar la imagen de marca no se percibe como una mala práctica para los stakeholders:

No creo que utilice campañas como La Marató para mejorar su imagen, no pienso que esta sea la finalidad, aunque seguro que acciones como esta mejoran su imagen (Ciudadanía, docente, 34 años).

Yo creo que indirectamente sí lo utiliza, y creo que es positivo, al final lo importante es sensibilizar y utilizar todas las vías de comunicación (Proveedores, gerente, 39 años).

Aunque hay muchas más respuestas que van por el mismo camino, se han extractado las que muestran más fehacientemente las opiniones de toda la cadena de valor.

\section{La Marató como acción de la RSC de TV3}

Las respuestas a esta pregunta, al igual que en el caso de la pregunta relacionada con la $\mathrm{RC}$, tienen un importante sesgo hacia quienes confirman que se trata de una acción de la RSC (tabla 7). 


\section{Tabla 7. La Marató como acción de la reputación social corporativa de TV3}

\begin{tabular}{|l|c|c|c|}
\hline \multicolumn{1}{|c|}{ Stakeholders } & $\begin{array}{c}\text { Sí } \\
\text { (cifras en porcentaje) }\end{array}$ & $\begin{array}{c}\text { No } \\
\text { (cifras en porcentaje) }\end{array}$ & NS/NR \\
\hline Empleados & 88.6 & 7.5 & 3.9 \\
\hline Clientes & 52.9 & 47.1 & 0.0 \\
\hline Proveedores & 83.8 & 9.6 & 6.6 \\
\hline Ciudadanía & 75.2 & 11.6 & 13.2 \\
\hline Organizaciones, instituciones y asociaciones & 67.3 & 23.6 & 9.1 \\
\hline Competencia y sector estratégico & 89.4 & 10.6 & 0.0 \\
\hline Instituciones públicas & 95.4 & 4.6 & 0.0 \\
\hline Promedio & 78.9 & 16.4 & 4.7 \\
\hline
\end{tabular}

Quienes están más seguros de que se trata de una acción de la RSC son las Instituciones públicas, y es comprensible que sea la Ciudadanía el stakeholder que más dudas tenga sobre esta pregunta, pues no todos los ciudadanos están familiarizados con el significado de la RSC. De esta forma, los grupos de interés vinculan de forma directa e indirecta La Marató a la RSC de TV3. Es decir, para algunos de los encuestados, La Marató es una forma de materializar las acciones de la RSC por parte de TV3, pero para otros no hace parte de una estrategia previamente concebida, sino que representa una obligación como empresa pública y una acción que, debido al tiempo que se viene desarrollando, está concebida como un aporte a la sociedad, como una acción social.

La trayectoria de La Marató influye en que muchos de los stakeholders perciban esta iniciativa como altruista, lo cual la desvincula de objetivos de marketing:

La Marató cumple los dos parámetros: la responsabilidad y la acción social. Pero por otro lado la RSC es un concepto muy nuevo y cuando TV3 comenzó a hacer La Marató no se sabía nada de la RSC (Organizaciones, instituciones y asociaciones, educadora social, 31 años).

Hoy en día, es el ejemplo más claro de la RSC. Cuando se ideó La Marató no se hablaba de la RSC y más de 20 años después es el mejor exponente (Empleados, licenciado, 49 años). 
La historia de La Marató se tiene en cuenta en el momento de evaluar no solo su pertinencia, sino su enfoque y poder de convicción. La RSC se entiende por parte de los stakeholders como una estrategia reciente, razón por la cual atribuyen a la La Marató otras funciones mucho más arraigadas en la función social. Inclusive esto ocasiona que muchos otros encuestados señalen que La Marató más que una acción de la RSC es una obligación de TV3 por su condición de medio de comunicación público:

Desconozco si entre los objetivos de la organización de La Marató se incluye la mejora de la imagen de TV3. Pero entenderíamos que esto es totalmente lícito, incluso consideramos que es una obligación de Ios máximos responsables de TV3 y de cualquier empresa (Organizaciones, instituciones y asociaciones, profesional, 67 años).

Al ser un medio de comunicación público, con programas, como La Marató, transmiten proximidad y se implican con la sociedad (Ciudadanía, administrativa, 32 años).

La función social de TV3 como medio de comunicación es el mayor argumento de la Ciudadanía para valorar La Marató como una obra dirigida al beneficio social más que a los propios menesteres de la marca. Es la Ciudadanía el grupo en el que más se evidencia la premisa de que TV3 tiene "obligaciones" con todos los grupos de interés, pero, sobre todo, con lo que le acontece a la comunidad.

Por su lado, los encuestados de las Organizaciones, instituciones y asociaciones admiten que La Marató cumple una doble función, ya que, aparte de la labor social expresada por la ciudadanía, le suman un beneficio directo en la marca:

Los objetivos dobles son muy interesantes, TV3 mejora la marca y, al mismo tiempo, hace responsabilidad social: todo un lujo (Organizaciones, instituciones y asociaciones, gerente, 40 años).

TV3 pone al servicio de las instituciones su imagen, infraestructura, contactos, etc., para la difusión de problemáticas y acciones concretas para solventarlas, y canaliza la posibilidad de tener ayudas económicas para apoyarlos (Organizaciones, instituciones y asociaciones, directora, 49 años). 
Como sucediera con la anterior pregunta, a los stakeholders no les parece una mala estrategia que un medio de comunicación social, como TV3, utilice acciones de la RSC para ganar o mejorar su imagen de marca; si ya lo hace como una acción social, los demás beneficios vendrán por añadidura.

\section{La incidencia de La Marató en la relación con los stakeholders}

La última pregunta de la encuesta intenta determinar si acciones de la RSC mejoran la relación con los grupos de interés (tabla 8).

\section{Tabla 8. Incidencia de La Marató en la relación con los stakeholders de TV3}

\begin{tabular}{|l|c|c|c|}
\hline \multicolumn{1}{|c|}{ Stakeholders } & $\begin{array}{c}\text { Sí } \\
\text { (cifras en porcentaje) }\end{array}$ & $\begin{array}{c}\text { No } \\
\text { (cifras en porcentaje) }\end{array}$ & NS/NR \\
\hline Empleados & 66.0 & 34.0 & 0.0 \\
\hline Clientes & 52.9 & 35.2 & 11.9 \\
\hline Proveedores & 38.7 & 54.8 & 6.5 \\
\hline Ciudadanía & 75.9 & 9.7 & 14.4 \\
\hline Organizaciones, instituciones y asociaciones & 58.2 & 18.2 & 23.6 \\
\hline Competencia y sector estratégico & 47.3 & 36.8 & 15.9 \\
\hline Instituciones públicas & 45.4 & 18.1 & 36.5 \\
\hline Promedio & 54.9 & 29.5 & 15.6 \\
\hline
\end{tabular}

La Ciudadanía es el grupo de interés que mejor valora la relación que genera La Marató. Lo que más valora es la labor de solidaridad, con la cual se sienten identificados y generen un vínculo más estrecho con TV3. Los Clientes de TV3 son un poco más parcos en el momento de responder, no obstante sus opiniones son igualmente positivas. En el caso de las Organizaciones, instituciones y asociaciones, se descubren tanto opiniones que admiten un beneficio como aquellas que no lo refieren.

Los Empleados son otro de los grupos de interés más prolíficos en las respuestas sobre la incidencia de La Marató en su relación con TV3. Las respuestas positivas establecen en su mayoría la oportunidad de trabajar de manera conjunta con personas de otros departamentos, razón por la cual mejora, según su visión, la relación con TV3. Es claro que en las respuestas se puede advertir que más que mejorar la relación de los Empleados con 
TV3 como empresa de comunicación lo que mejoran son las relaciones interpersonales entre los trabajadores.

En la Competencia y sector estratégico, se pueden reseñar respuestas muy positivas. En general, aseguran que La Marató es una iniciativa interesante y que genera cohesión entre profesionales. Es cierto que el día en que se celebra La Marató muchos otros medios de comunicación colaboran difundiendo aspectos centrales del evento, por varios motivos, pero, en especial, porque hacen una cobertura como noticia o porque también sienten la necesidad de aportar.

Esta solidaridad de los medios de comunicación también es una de las razones por las que se considera una buena herramienta para mejorar las relaciones con las Instituciones públicas, en que el servicio a los demás es el punto de anclaje más sobresaliente:

Sí. Trabajar conjuntamente en una acción solidaria como La Marató mejora los lazos y los vínculos entre todos los participantes (Instituciones públicas, técnica, 48 años).

Es trascendental poder recopilar todas las opiniones de los grupos de interés, pues en muchas ocasiones se ha palpado cómo la simple respuesta afirmativa o negativa resuelve muy pocas situaciones que rodean a acciones tan complejas como la RSC de una empresa de comunicación.

\section{Conclusiones}

Si el objetivo fundamental de este artículo es conocer si comunicar la RSC revierte en la RC, la respuesta es concluyente si nos ceñimos a la valoración de los stakeholders: $93 \%$ de los encuestados considera que hay una incidencia positiva en la RC de TV3. Es posible inclusive asegurar que toda la cadena de valor tiene una percepción muy positiva de La Marató y de las acciones de la RSC que TV3 implementa.

Pero los mismos stakeholders que definen que La Marató beneficia la RC creen que esta iniciativa no mejora la imagen de marca. Esto resultó contradictorio hasta que pudo dilucidarse al analizar las valoraciones de los 
stakeholders a través de las preguntas abiertas. Este es un caso muy interesante, pues los grupos de interés ya tienen una buena imagen de La Marató y de TV3, y sus respuestas cualitativas están más enfocadas en describir que no consideran que TV3 emprenda estas acciones con un objetivo mercantilista de mejorar la imagen de la marca, inclusive asumiendo que si fuera así a muchos no les molestaría.

Asimismo, se concluye cómo la cadena de valor percibe en su gran mayoría que la gestión que hace TV3 con La Marató es transparente. Esto es trascendental si se valora la importancia que le otorgan los stakeholders a las empresas transparentes, máxime cuando se conocen escándalos de corrupción en todo tipo de organizaciones. Es significativo que sean los grupos de interés "internos" (empleados, clientes y proveedores) quienes mejor califican la transparencia de TV3, siendo los stakeholders los que conocen directamente la gestión que realiza TV3 con La Marató.

En contraposición, para los stakeholders no es claro que La Marató contribuya a mejorar la gestión empresarial de TV3. Incluso se descubre una posición muy crítica de los empleados al considerar que La Marató no favorece la gestión empresarial de TV3. Es decir, en este caso, aunque consideran La Marató como transparente, no la contemplan como una iniciativa que genere beneficio en la gestión de TV3.

Se puede concluir, además, que los grupos implicados consideran claramente La Marató como una acción de la RSC. La respuesta es categórica en todos los grupos de interés, lo cual desmitifica la idea de algunos autores (Viñarás, 2010; Korin, 2011; Vives, 2011; Fernández, 2013) sobre la falta de conocimiento por parte de muchos colectivos sobre lo que es la RSC y su desarrollo.

Poder evaluar las percepciones que sobre la RC tiene toda la cadena de valor igualmente admite diversos enfoques. La variedad de grupos implicados hace que según sea su relación con TV3 se obtengan conclusiones diferenciadas. Quienes son más incisivos en sus críticas y ataques son los stakeholders que se asume tienen menos información sobre TV3 (Ciudadanía), 
en contraposición de aquellos colectivos que, además de exaltar la gestión de La Marató, se atreven a realizar sugerencias y proponer posibles soluciones (Empleados y Proveedores).

No obstante que otros investigadores hayan concluido que algunos stakeholders pueden tener reticencias respecto de las acciones de la RSC, en esta investigación se puede percibir que la cadena de valor recibe con agrado la acción de la RSC de La Marató, especialmente porque consideran que TV3 demuestra sensibilidad institucional ante la problemática social. La sensibilidad de una organización puede ser un factor trascendental y diferenciador en momentos en los que los grupos de interés perciben a muchas empresas como entidades sin valores y que solo responden a sus intereses económicos.

La idea de utilizar la RSC para beneficios relacionados con el marketing no es un aspecto que los stakeholders hayan destacado. Aunque pocos consideren que la RSC que desarrolla TV3 busca pauta publicitaria o mejorar su imagen, la mayoría de los grupos asumen La Marató como parte integral de la marca. Esta posición aleja las dudas del imaginario colectivo sobre el uso de La Marató con fines mercantilistas, ya que según la propia cadena de valor TV3 no requiere acciones de la RSC para aumentar su capital de reputación.

La continuidad con la que TV3 lleva realizando La Marató (veintitrés años) es un elemento diferenciador al evaluar las percepciones de los stakeholders. Es decir, ya que los grupos de interés asumen La Marató como una acción que perdura en el tiempo y que tiene una base sólida, no la perciben como una labor paliativa o coyuntural. La RSC no es por sí sola la única condición para generar reputación e imagen de marca. Los stakeholders interpretan La Marató como una acción de la RSC, pero le suman dos razones para una excelente valoración: que sea una buena causa (trabajar por el bien común) y que las cosas se hagan bien hechas (apuesta por la calidad). En este aspecto, se reitera que, aunque expresen que TV3 recibe beneficios en cuanto a su imagen por parte de La Marató, consideran que no lo hace con ese único objetivo y que sus expectativas y prioridades van más dirigidas a cumplir objetivos relacionados con la filantropía. 
Aunque se encuentra un importante grado de concordancia en las opiniones y percepciones de los grupos de interés, evaluar la RC por separado dentro de la cadena de valor permite obtener diferentes conclusiones. Incluso, los empleados asocian las acciones que realizan con La Marató con una oportunidad para estrechar los vínculos con otros empleados o sentir que hacen parte activa de las iniciativas de la RSC. De esta afirmación se puede sacar una conclusión interesante que podría servir de ejemplo a otras empresas, pues dar la oportunidad para que más grupos de interés participen en las acciones de la RSC puede reportar a las organizaciones un beneficio adicional. No solo permite mejorar las relaciones interpersonales, sino que, además, hace que dichos colectivos tengan sentido de pertenencia con las iniciativas de la RSC y con la empresa.

TV3 realiza esfuerzos para comunicar a todos los stakeholders cómo invierte los fondos recaudados cada año en La Marató. Se utilizan varias plataformas para la rendición de cuentas; sin embargo, hay diferencias marcadas en relación con la recepción de dicha información. Se evidencia que empleados, proveedores, clientes, competencia y sector estratégico son los que más conocimiento tienen de los esfuerzos en accountability. Las empresas que desean poner en marcha campañas de rendición de cuentas requieren diversidad de medios y una mejor estrategia de comunicación.

La comunicación de la RSC genera impactos decisivos en la RC de las organizaciones, sean positivos, sean negativos. La marca, como parte fundamental de los activos empresariales, es quizá el elemento sobre el que más influencia se ejerce. La RC de La Marató está respaldada por la reputación que viene forjando TV3 desde hace treinta años, pero también se evidencia que existe una relación de mutuo beneficio, pues la marca TV3 ha recibido un remanente importante de imagen y de la $\mathrm{RC}$ que le sirve de reservorio para afrontar posibles crisis. TV3 como marca no solo ha sabido aprovechar los beneficios de la comunicación de la RSC, sino que, además, la ha incorporado a su $\mathrm{ADN}$ corporativo. Esta forma de afrontar la RSC le permite obtener reconocimiento y valoración de todos sus stakeholders.

Con la excelente valoración de TV3, los grupos de interés no solo vinculan la marca con La Marató como acción de la RSC, sino con otras 
iniciativas de la RSC que se llevan a cabo, por ejemplo, campañas publicitarias gratuitas a organizaciones no gubernamentales (ONG). De esta forma, TV3 ha generado una percepción de organización responsable y marca cercana. A pesar de los aspectos positivos mencionados, TV3 debe desarrollar estrategias en algunos frentes de trabajo donde tiene aún desventajas. Deberá redoblar esfuerzos en la rendición de cuentas, pues, aunque ya la realizan, no impactan a todos los grupos de interés de igual forma. Dada la heterogeneidad de los stakeholders de TV3, la marca deberá reforzar diferentes formas de comunicar el destino del dinero recaudado, las instituciones beneficiadas y los logros alcanzados.

Con el caso estudiado, y para ese contexto, se puede afirmar que la comunicación de la RSC genera destacables beneficios en la RC de las empresas de comunicación, sin distinción de cuáles sean los vínculos que tenga con cada uno de sus grupos de interés.

\section{Referencias}

Aaker, D. (2002). Construir marcas poderosas. Barcelona: Gestión.

Alloza, A. (2005). La reputación corporativa, la eficacia de la comunicación, la marca y la creación de valor. Investigación y Marketing, 86, 6-15.

Alsop, R. (2004). The 18 Immutable Laws of Corporate Reputation: Creating, Protecting \& Repairing your most Valuable Asset. Londres: Kogan Page.

Álvarez, J. T. (2008). Entorno mixto para gestión de reputación e intangibles. En M. Fernández (ed.), Comunicación en la sociedad red: la construcción mediática de la realidad (pp. 73-95). Ávila: Universidad Católica de Ávila.

Arendt, S. y Brettel, M. (2010). Understanding the Influence of Corporate Social Responsibility on Corporate Identity, Image, and Firm Performance. Management Decision, 48(10), 1469-1492. 
Argenti, P. y Forman, J. (2002). The Power of Corporate Communication. Nueva York: McGraw-Hill.

Bigné, E., Currás, R. y Aldás, J. (2012). Dual Nature of Cause-Brand Fit: Influence on Corporate Social Responsibility Consumer Perception. European Journal of Marketing, 46(3-4), 575-594.

Borrini, A. (2006). Publicidad, diseño y empresa. Buenos Aires: Infinito.

Capriotti, P. (2007). La responsabilidad social corporativa de las empresas españolas en los medios de comunicación. Zer, 23, 61-74.

Capriotti, P. (2009). Branding corporativo: fundamentos para la gestión estratégica de la identidad corporativa. Santiago de Chile: Andros.

Castaldo, S., Perrini, F., Misani, N. y Tencati, A. (2009). The Missing Link between Corporate Social Responsibility and Consumer Trust: The Case of Fair Trade Products. Journal of Business Ethics, 84, 1-15. doi.org/10.1007/s10551-008-9669-4

Cerviño, J. (2002). Marcas internacionales: cómo crearlas y gestionarlas. Madrid: Pirámide.

Charlo, M., Moya, I. y Muñoz, A. (2013). Factores diferenciadores de las empresas del índice de responsabilidad español. Cuadernos de Gestión, 13(2), 15-37.

Costa, R. y Menichini, T. (2013). A Multidimensional Approach for CSR Assessment: The Importance of the Stakeholder Perception. Expert Systems with Applications, 40, 150-161. doi.org/10.1016/j. eswa.2012.07.028

Delgado, J., Quevedo, E. y Díez, J. (2013). The Impact of Corporate Reputation on Firm Risk: A Panel Data Analysis of Spanish Quoted Firms. British Journal of Management, 24, 1-20. 
Doane, D. (2010). Una perspectiva alternativa acerca de las marcas: los mercados y la moral. En G. Brujó (ed.), En clave de marcas (pp. 248259). Madrid: LID.

Doorley, J. y García, H. (eds.) (2011). Reputation Management: The Key to Successful Public Relations and Corporate Communication. Nueva York: Routledge.

Dowling, G. (2001). Creating Corporate Reputations: Identity, Image, and Performance. Nueva York: Oxford University Press.

Du, S., Bhattacharya, C. y Sen, S. (2010). Maximizing Business Returns to Corporate Social Responsibility (CSR): The Role of CSR Communication. International Journal of Management Reviews, 12(1), 8-19. doi.org/10.1111/j.1468-2370.2009.00276.x

Earl, S. y Waddington, S. (2012). Brand Anarchy: Managing Corporate Reputation. Londres: Bloomsbury.

Epstein, M. y Birchard, B. (2001). La empresa honesta: cómo convertir la responsabilidad corporativa en ventaja competitiva. Barcelona: Paidós.

Fernández, T. (2013). Las corporaciones de radiotelevisión pública de la Unión Europea y el incipiente uso de la web 2.0 para comunicar RSC. Journal of Communication, 6, 86-110.

Ferré Pavia, C. y Orozco, J. (2011).El diálogo entre empresas de comunicación y sus stakeholders en América y Europa: valores de la cultura corporativa en 40 informes de RSC al Pacto Mundial. Cuadernos de Información, 29, 91-104.

Firestein, P. (2009). Crisis of Character: Building Corporate Reputation in the Age of Skepticism. Nueva York: Union Square Press.

Fombrun, C. (1996). Reputation: Realizing Value from the Corporate Imagen. Boston: Harvard Business School Press. 
Freeman, B. (2006). Substance Sells: Aligning Corporate Reputation and Corporate Responsibility. Public Relations Quaterly, 51(1), 12-19.

Fundació La Marató de TV3 (2015). Totes les maratons. Recuperado de http://www.tv3.cat/marato/totes_les_maratons.htm

García, F. (2002). La reputación como recurso estratégico: un enfoque de recursos y capacidades (Tesis de Doctorado, Universidad de La Laguna). Recuperado de ftp://tesis.bbtk.ull.es/ccssyhum/cs133.pdf

Hannington, T. (2006). Cómo medir y gestionar la reputación de su empresa. Barcelona: Deusto.

Helm, S. y Tolsdorf, J. (2013). How Does Corporate Reputation Affect Customer Loyalty in a Corporate Crisis? Journal of Contingencies and Crisis Management, 21(3), 144-152. doi.org/10.1111/14685973.12020

Hillenbrand, C., Money, K. y Ghobadian, A. (2013). Unpacking the Mechanism by which Corporate Responsibility Impacts Stakeholder Relationships. British Journal of Management, 24, 127-146. doi. org/10.1111/j.1467-8551.2011.00794.x

Ingenhoff, D. y Koelling, M. (2012). Media Governance and Corporate Social Responsibility of Media Organizations: An International Comparison. Business Ethics: A European Review, 21(2), 154-167.

Jackson, K. (2004). Building Reputational Capital: Strategies for Integrity and Fair Play that Improve the Bottom Line. Nueva York: Oxford University Press.

Kim, H. (2011). A Reputational Approach Examining Publics Attributions on Corporate Social Responsibility Motives. Asian Journal of Communication, 21(1), 84-101. doi.org/10.1080/01292986.2 010.524230 
Korin, M. (2011). Los medios de comunicación. En A. Vives y E. Peinado (eds.), La responsabilidad social de la empresas en América Latina (pp. 185-202). Washington: Cumpetere.

Laczniak, G. y Murphy, P. (2012). Stakeholder Theory and Marketing: Moving from a Firm-Centric to a Societal Perspective. Journal of Public Policy \& Marketing, 31(2), 284-292. doi.org/10.1509/jppm.10.106

Lange, D., Lee, P. y Dai, Y. (2011). Organizational Reputation: A Review. Journal of Management, 37(1), 153-184. doi. org/10.1177/0149206310390963

Larkin, J. (2003). Strategic Reputation Risk Management. Nueva York: Palgrave MacMillan.

Lee, E., Park, S. y Lee, H. (2013). Employee Perception of CSR Activities: Its Antecedents and Consequences. Journal of Business Research, 66, 1716-1724. doi.org/10.1016/j.jbusres.2012.11.008

Lindgreen, A., Xu, Y., Maon, F. y Wilcock, J. (2012). Corporate Social Responsibility Brand Leadership: A Multiple Case Study. European Journal of Marketing, 46(7-8), 965-993.

Mainwaring, S. (2011). We First: How Brands and Consumers Use Social Media to Build a Better World. Nueva York: Palgrave.

Martínez, P., Pérez, A. y Rodríguez, I. (2014). CSR Influence on Hotel Brand Image and Loyalty. Academia Revista Latinoamericana de Administración, 27(2), 267-283. doi.org/10.1108/ARLA-12-2013-0190

Miralles, J. (2006). Ética empresarial y RSC: ¿Complementariedad? ¿Diálogo? En J. Benavides, J. Fernández y N. Villagra (eds.), La ética y la responsabilidad social de las empresas y organizaciones (pp. $77-$ 96). Madrid: Universidad Pontificia Comillas. 
Mishina, Y., Block, E. y Mannor, M. (2012). The Path Dependence of Organizational Reputation: How Social Judgment Influences Assessments of Capability and Caracter. Strategic Management Journal, 33, 459-477. doi.org/10.1002/smj.958

Öberseder, M., Schlegelmilch, B. y Murphy, P. (2013). CSR Practices and Consumer Perceptions. Journal of Business Research, 66, 1839-1851. doi.org/10.1016/j.jbusres.2013.02.005

Olabe, F. (2012). La RSC como factor de reputación en los clubes de fútbol profesionales. Revista Internacional de Investigación en Comunicación AdResearch ESIC, 6(6), 144-157.

Olcese, A. (2011). Creación de valor y responsabilidad social de la empresa en las empresas del Ibex 35. Barcelona: RACEF.

Orozco, J. y Ferré Pavia, C. (2011). Los stakeholders de las empresas de comunicación en el ámbito de la responsabilidad social corporativa. Folios, 25, 107-125.

Orozco, J. y Roca, D. (2011). Construcción de imagen de marca y reputación a través de campañas publicitarias de RSC. Sphera Publica, 11, 273-289.

Pérez Serrano, M. J. (2010). Análisis del valor de las empresas informativas. Palabra Clave, 13(1), 47-58.

Preciado-Hoyos, A. (2015). Apoyo de las relaciones públicas a los programas de responsabilidad social en las empresas del sector eléctrico colombiano. Palabra Clave, 18(1), 239-257. doi.org/10.5294/ pacla.2015.18.1.10

Quevedo, E. (2003). Reputación y creación de valor. Madrid: Thomson.

Ráfols, C. (2007). ¿Ética en las empresas o empresas éticas? Avanzar en una implantación contradictoria. En R. Alcoberro (ed.), Ética, economía 
y empresa: la dimensión moral de la economía (pp. 183-192). Barcelona: Gedisa.

Ramiro, P. (2009). Las multinacionales y la responsabilidad social corporativa: de la ética a la rentabilidad. En J. Hernández y P. Ramiro (ed.), El negocio de la responsabilidad: critica de la responsabilidad social corporativa de las empresas transnacionales (pp. 47-78). Barcelona: Icaria.

Saeedniaa, H. y Sohanib, Z. (2013). An Investigation on the Effect of Advertising Corporate Social Responsibility on Building Corporate Reputation and Brand Equity. Management Science Letters, 3, 11391144. doi.org/10.5267/j.msl.2013.03.012

Sánchez, A. (2001). El comportamiento ético en las empresas de comunicación. En M. Codina (ed.), De la ética desprotegida: ensayos sobre deontología de la comunicación (pp. 21-39). Navarra: Eunsa.

Sanz de la Tajada, L. (1994). Integración de la identidad y la imagen de la empresa. Madrid: ESIC.

Sanz de la Tajada, L. (1996). Auditoría de la imagen de empresa: métodos y técnicas de estudio de la imagen. Madrid: Síntesis.

Simic, P. y Belliu, A. (2001). Corporate Social Responsibility and CauseRelated Marketing: An Overview. International Journal of Advertising, 20, 207-222.

Tang, Z., Hull, C. y Rothenberg, S. (2012). How Corporate Social Responsibility Engagement Strategy Moderates the CSR-Financial Performance Relationship. Journal of Management Studies, 49(7), 1274-1303. doi.org/10.1111/j.1467-6486.2012.01068.x

Torelli, C., Monga, A. y Kaikati, A. (2012). Doing Poorly by Doing Good: Corporate Social Responsibility and Brand Concepts. Journal of Consumer Research, 38(5), 948-963. 
Van Riel, C. (1997). Comunicación corporativa. Madrid: Prentice Hall.

Villagra, N. (2007). ¿Por qué tenemos miedo de comunicar la responsabilidad social corporativa? En A. Bajo y N. Villagra (eds.), Evolución conceptual y práctica de una gestión responsable (pp. 77-91). Madrid: Universidad Pontificia Comillas.

Viñarás, M. (2010). El discurso de la RSC en los medios de comunicación social. Vivat Academia, 110, 90-104.

Vives, A. (2011). Mirada crítica a la responsabilidad social de la empresa en Iberoamérica. Washington: Cumpetere. 